Luiz Homero L. Martins

luizunb@pop.com.br

Júlio Cesar T. Ferro

jct.ferro@gmail.com

Jorge Luiz A. Ferreira

jorge@unb.br

José Alexander Araújo

Member, $A B C M$

alex07@unb.br

University of Brasilia

Department of Mechanical Engineering

70910-900 Brasília, DF. Brazil

Lucas Susmel

ssı@unife.it

Trinity College

Dept. of Mechanical Engineering

Dublin 2, Ireland or

University of Ferrara, Dept. of Engineering

Ferrara. Italy

\section{A Notch Methodology to Estimate Fretting Fatigue Strength}

The aim of this work is to propose a methodology to estimate the fatigue limit of cylindrical contacts under a partial slip regime. Taylor's point stress method, usually applied to estimate fatigue limit for notched structures, was associated with the Modified Wöhler Curves to define the fretting crack initiation threshold methodology. Twenty-nine tests on cylindrical contacts were selected from the literature and considered to evaluate the quality of the estimates. The results agree well for twenty-three experimental data. As the fatigue limit under fully reversed bending is the fatigue parameter usually available for most metallic alloys, it was also showed how the second fatigue limit needed to calibrate the proposed procedure could be estimated by taking full advantage from other standard predictive methodologies previously devised to estimate the mean stress effect under uniaxial fatigue loading.

Keywords: fretting fatigue, notch fatigue, multiaxial fatigue, short cracks, critical distance, size effect.

\section{Introduction}

Fretting fatigue occurs at the contact interface of mechanical joints, which experience some sort of relative movement due to vibration. The rubbing of tightly fitting joints gives rise to a small scale wear mechanism denoted fretting. This damage of the surfaces together with the stress concentration produced by the contact region usually speed up the nucleation and early growth of fatigue cracks eventually leading to a premature failure, should at least one of the components of the assembly be subjected to a remote fatigue load.

The estimation of fretting fatigue strength is particularly important in safety-critical applications such as occur in the aerospace or nuclear industries. The designer needs to know how well the interface will perform under the imposed loading conditions and, in particular, whether either of the contacting components is likely to fail during the operating lifetime or inspection interval. This is a complex problem, since there are a number of coupled phenomena present. The imposed load may, itself, depend on the interface response, in particular the level of frictional damping present. Friction coefficients may vary with position and with time, and wear may lead to changes in geometry and contact tractions as well as removing initiated cracks.

It is generally accepted that fretting can play a part in accelerating crack initiation and short crack growth, probably due to the presence of a high but extremelly localized stress gradients. Fouvry et al. (2002) found that, applying a multiaxial model to Hertzian contact of a sphere on a flat produced an under-estimate of specimen life unless the fatigue parameter was averaged over a characteristic volume. The need for this averaging process was ascribed to the high stress gradients present. The material cracking behaviour under fretting fatigue can be assumed to be similar to that occurring in notched components under "conventional" fatigue loading: crack initiation, and its initial growth, depends on the distribution of the entire stress field damaging the fatigue process zone (Araujo et al, 2004, 2006; Valleano et al, 2004). In particular, according to the experimental results published by Vallellano et al. (2004) and generated by testing Al 7075-T6 sphere-plane contacts, initiation and early growth of cracks occur at small angles to the surface. Subsequently, cracks change their direction to grow along a line almost perpendicular to the contact zone surface. Finally, it is interesting to highlight that when failures do not occur in the highcycle fatigue regime, it is usual to find cracks which were arrested by the first grain boundary (as it happens to plain metal specimens under conventional fatigue loading). Attempts have been made to predict fretting fatigue thresholds using short crack methods (Araujo and Nowell, 1999). Unfortunately, it is well known that to correctly model short crack behaviour, linear-elastic approaches may not be adequate.

The aim of this paper is to estimate the fretting fatigue strenght of an Aluminium alloy by considering a notch methodology based on the theory of Critical Distances (Susmel et al, 2004; Taylor 1999) and a high-cycle multiaxial fatigue model (Susmel and Taylor, 2003; Susmel, 2004). The application of such method requires the determination of two fatigue limits under different load conditions. As the S-N curve for fully reversed bending is usually the fatigue information available for most mettalic alloys it was also the aim of this work to show the influence of estimating another fatigue limit on the predictive methodology.

Paper accepted July, 2006. Technical Editor: Paulo E. Miyagi. 


\section{Nomenclature}

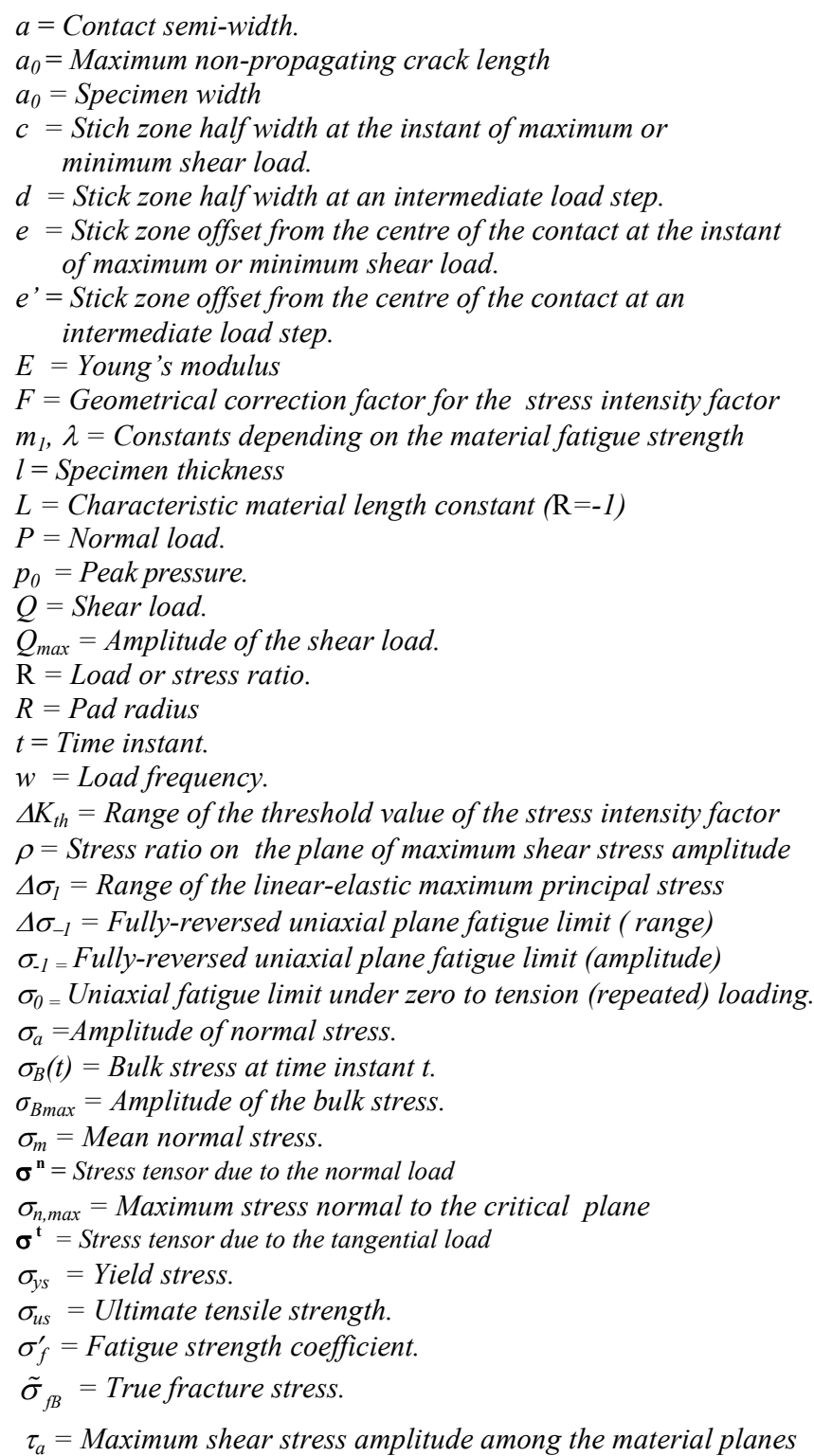

\section{Fatigue Damage and Structural Volume}

The use of the Modified Wöhler Curve Method (MWCM) in terms of the Theory of Critical Distances (TCD) is based on the assumption that all the physical processes leading to crack initiation are confined within the so-called structural volume. The size of this volume is assumed not to be dependent on either the stress concentration feature weakening the component or the complexity of the stress field damaging the fatigue process zone (Susmel, 2004).

To define the size of this volume, consider an infinite plate with a central through-crack (Fig. 1a). This plate is subjected to a remote fully-reversed uniaxial fatigue loading $(\mathrm{R}=-1)$. Observing that, thanks to the above assumptions, geometry, nominal stress gradient and non-zero mean stress do not affect the crack growth, the above configuration can be assumed to be representative of the "pure" material cracking behaviour. In other words, when an infinite plate is weakened by a central crack, the geometrical correction factor for the LEFM stress intensity factor, $F$, is equal, by definition, to unity. This means that, according to the conventions the LEFM theory is based on, the crack propagation is not influenced by the geometry of the component. Moreover, the fact that the reference cracked plate is subjected to a remote fully-reversed tension-compression loading results in two important consequences: the crack propagation phenomenon is not affected by a nominal stress gradient (as, for instance, it would happen if the plate was subjected to a remote bending moment) as well as by the presence of non-zero mean stress. As to the mean stress effect, the idea is that an efficient multiaxial fatigue criterion should be able to take into account this phenomenon even if calibrated by using material fatigue properties generated under a load ratio, $R$, equal to -1 . All the above simplifying assumptions were formed in order to coherently reinterpret the MWCM in terms of TCD.

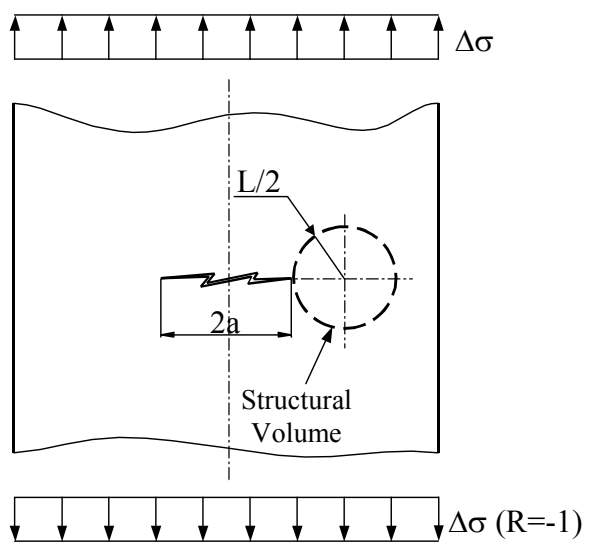

(a)

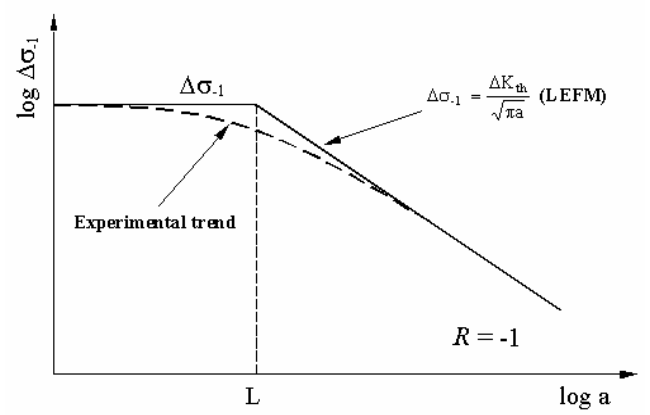

(b)

Figure 1 (a) Central through-crack in an infinite plate subjected to a remote uniaxial load and (b) Kitagawa and Takahashi's diagram.

Consider now the classical Kitagawa-Takahashi curve by approximating it to the two straight asymptotic lines plotted in Fig. 1b: the horizontal line corresponds to the plain fatigue limit, whereas the slopping one is plotted according to the Linear Elastic Fracture Mechanics (LEFM). The length at which these two lines intersect each other turns out to be:

$$
L=\frac{1}{\pi}\left(\frac{\Delta K_{t h}}{\Delta \sigma_{-1}}\right)^{2}
$$

In the above equation, $\Delta \sigma_{-1}$ is the plane fatigue limit range and $\Delta K_{t h}$ is the range of the threshold value of the stress intensity factor (both determined under load ratios, $R$, equal to -1). According to the fact that the material characteristic length $L$ is defined by two material properties, it is evident that $L$ turns out to be a material property which is different for different materials (Taylor 1999).

Observing now the trend schematised by the two straight asymptotic lines in Kitagawa-Takahashi's diagram, it is possible to 
assume that as long as the half-length of the crack is lower than $L$, no reduction of the nominal fatigue limit occurs. Therefore, the size of the structural volume can be considered to be directly related to the material characteristic length, $L$. To be precise, in order to avoid a reduction of the nominal fatigue limit, all the cracking processes must be confined within this area, which can be supposed to be circular in 2D bodies and spherical in 3D components (Susmel, 2004) (Fig. 1a).

It is possible to observe now that when a component is in the fatigue limit condition some micro/meso-cracks are always present within the structural volume, and it holds true independently of the stress concentration feature weakening the component (Akiniwa et al, 2001). In particular, it is important to remember that the sharper the notches are then the longer the length of non-propagating cracks. When plain specimens are in the fatigue limit condition, the crack propagation is arrested either by the first grain boundary or by the first micro-structural barrier (Miller, 1993). On the contrary, in the presence of sharp notches, the maximum length of non-propagating cracks is equal to (Yates and Brown, 1987):

$$
a_{0}=\frac{1}{\pi}\left(\frac{\Delta K_{t h}}{F \Delta \sigma_{-1}}\right)^{2}
$$

where $\mathrm{F}$ is the geometrical correction factor for the LEFM stress intensity factor.

If non-propagating cracks emanate from the tip of a notch weakening a real component, $\mathrm{F}$ is always larger than unity (Tada et al, 2000). This makes it evident that non-propagating cracks are always confined within the structural volume, even when they reach their maximum length.

All the above arguments seem to strongly support the idea that the fatigue process zone has a size which is directly related to the material characteristic length, $L$. The question now is "What is the material cracking behaviour within the fatigue process zone?". During the last few years, we extensively investigated crack paths within the structural volume in the high cycle fatigue regime. In particular, we considered specimens of steel weakened by different geometrical feature and subjected to both uniaxial and biaxial fatigue loading (Susmel at al, 2003; Menghetti et al, 2004; Susmel and Taylor, 2004a).

Our understanding of the phenomenon is that initiation and initial growth of micro/meso-cracks are always mixed-mode governed and this process can be considered to be similar to the classical Stage 1 taking place in plain specimens (Miller, 1993). In particular, independently of stress concentration feature and degree of multiaxiality of the stress field damaging the fatigue process zone, crack initiation is mixed-mode dominated and the length of the Stage 1-like crack is equal to about $L / 2$. To be precise, the transition from a Stage 1-like to a Stage 2-like process occurs at a distance from the notch tip depending on the material characteristic length, $L$. When the crack length is larger than about $L / 2$, cracks tend to orient themselves in order to experience the maximum Mode I loading (Stage 2-like process). Therefore, if crack initiation is assumed to be the most important stage in determining fatigue limits, it is logical to believe that the critical plane approach is the soundest method to model the physical reality.

\section{The theory of critical distances (TCD) and the modified Wöhler curve method (MWCM)}

In the recent past, Taylor (1999) has proposed a new reinterpretation of the TCD to predict the uniaxial fatigue limit of components weakened by any kind of stress concentration feature. This approach postulates that the reference stress to be used to assess notched components can be calculated in different ways. In particular, it can be determined at a certain distance from the apex of the stress concentrator (Point Method, PM), it can be averaged along a line (Line Method, LM), or, finally it can be averaged over an area (Area Method, AM). The TCD was seen to be capable of predictions falling within an error interval of about $20 \%$ (Susmel and Taylor, 2004b). This method is essentially empirical, but Taylor (2001) has proposed that the LM may be related to the conditions for propagation of a notch-root crack of length $2 L$. Unfortunately, this would only justify the use of the method for sharp notches, giving no explanation as to the reason why the TCD is also successful in predicting fatigue limits in the presence of blunt notches.

In order to formalise the TCD in terms of the PM, consider a notched specimen subjected to a remote uniaxial fatigue loading (Fig. 2a). A notched component is in the fatigue limit condition if the range of the maximum principal stress at a distance from the notch tip equal to $L / 2$ equals the plain fatigue limit, $\sigma_{-1}$ (Taylor, 1999). In other words (see Fig. 2 for the symbolism definition):

$$
\frac{\Delta \sigma_{1}}{2}\left(r=\frac{L}{2}, \theta=0\right)=\sigma_{-1},
$$

where $L$ is given by Eq. (1). It is important to highlight here that, to properly apply the TCD, $L$ must always be determined for the correct load ratio, R (Taylor, 1999; Susmel and Taylor, 2003).

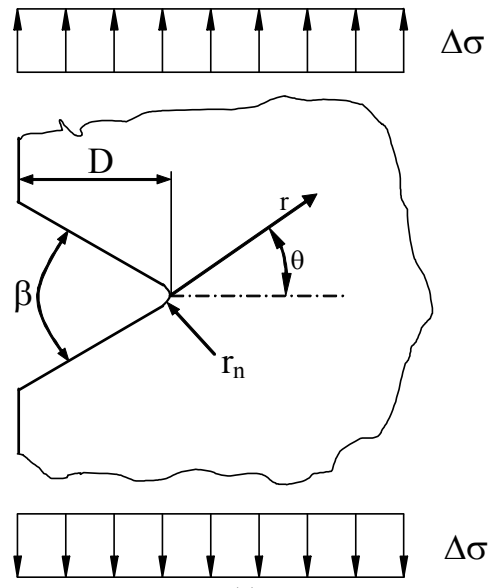

(a)

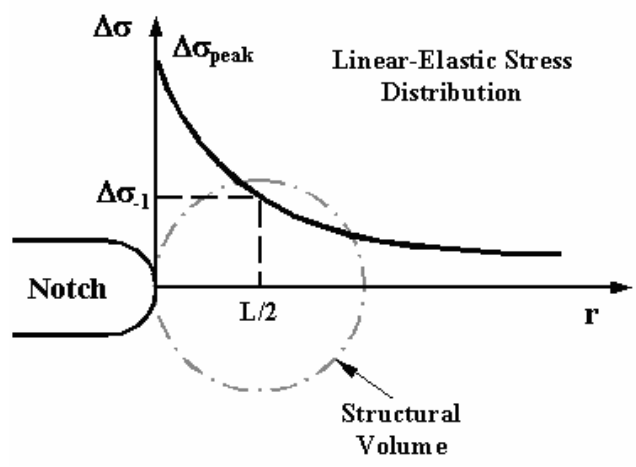

(b)

Figure 2. (a) Notched component subjected to a remote uniaxial fatigue loading; (b) Stress-Distance curve and Point Method.

As shown by Fig. 2b, according to the PM, the point at which the reference stress must be calculated exactly corresponds to the centre of the structural volume. 
The MWCM takes as its starting point the assumption that crack initiation is Mode II dominated, and it holds true independently of both stress concentration feature and degree of multiaxiality of the stress filed damaging the fatigue process zone. The MWCM formalised to assess components in the high-cycle fatigue regime turns out to be (Susmel and Lazzarini, 2002):

$$
\tau_{a}+m_{1} \frac{\sigma_{n, \max }}{\tau_{a}}-\lambda=0
$$

In the above equation, $\tau_{a}$ is the shear stress amplitude relative to the plane experiencing the maximum shear stress amplitude (critical plane), $\sigma_{\mathrm{n}, \max }$ is the maximum stress perpendicular to this plane and, finally $\lambda$ and $m_{l}$ are material constants that can be obtained from the fatigue limits generated under different loading conditions. In terms of the fatigue limits $\sigma_{-1}$ and $\sigma_{0}$ for tests under fully-reversed $(\mathrm{R}=-1)$ and repeated $(\mathrm{R}=0)$ uniaxial load, respectively, these constants will be:

$$
\begin{aligned}
& m_{1}=\frac{\sigma_{-1}-\sigma_{0}}{2}, \\
& \lambda=\sigma_{-1}-\frac{\sigma_{0}}{2}
\end{aligned}
$$

For most metallic alloys the fatigue parameter usually available is the fatigue limit under fully reversed uniaxial loading. To estimate $\sigma_{0}$ it is necessary to invoke an appropriate model capable to account for the effect of a mean direct stress on the fatigue endurance of the material, as the ones proposed by Smith et al (1970) (eq. 6), Goodman (1919) (eq.7 ) or Morrow (1968) (eqs. 8 and 9)) .

$$
\begin{aligned}
& \sigma_{0}=\frac{\sqrt{2}}{2} \sigma_{-1}, \\
& \sigma_{0}=\sigma_{-1}\left(1-\frac{\sigma_{0}}{\sigma_{u s}}\right), \\
& \sigma_{0}=\sigma_{-1}\left(1-\frac{\sigma_{0}}{\tilde{\sigma}_{f B}}\right), \\
& \sigma_{0}=\sigma_{-1}\left(1-\frac{\sigma_{0}}{\sigma_{f}^{\prime}}\right) .
\end{aligned}
$$

Being $\sigma_{u s}$ the ultimate tensile strength, $\sigma_{f}^{\prime}$ the fatigue strength coefficient and $\tilde{\sigma}_{\beta B}$ the true fracture stress.

The fact that Eq. (4) can be calibrated by using a plane fatigue limit and a material static property makes the MWCM suitable for being used by engineers engaged in assessing real components by reducing time and costs of the design process: unfortunately, it is well-known that in the industrial reality, too often, due to the lack of resources as well as of time, fatigue assessment has to be performed by using just few pieces of experimental information for the calibration of the criterion used to predict fatigue damage. It is worth mentioning that we have recently investigated in deep the accuracy of the MWCM in predicting the mean stress effect in fatigue (Susmel et al, 2005; Meneghetti et al, 2004). These systematic investigations proved that our method is successful in accounting for the presence of non-zero mean stresses, both under uniaxial and under multiaxial fatigue loading, even if calibrated by using fatigue properties generated under fully-reversed cyclic stress.
In light of these interesting results, eqs. (6)-(9) have been reported above to better show another important peculiarity of the approach employed in this study to address the fretting fatigue problem: the MWCM can successfully be calibrated also by estimating the needed material constants by taking fully advantage from wellknown and sound approaches devised in the past to predict the mean stress detrimental effect in the presence of conventional uniaxial fatigue loading.

For sake of simplification eq. (4) can also be expressed in terms of an error index (Susmel and Taylor, 2003) defined as:

$$
S U=\frac{\tau_{a}}{\lambda-m_{1} \frac{\sigma_{n, \max }}{\tau_{a}}}-1
$$

A negative value for $S U$ indicates that the dynamic solicitation is under the material multiaxial fatigue endurance, hence it would, in theory, last forever; or interpreted from a different point of view its also an indication that the component dimensions could be reduced up to the limiting state defined by $S U=0$. Reliability and accuracy of such model have been extensively checked both in the high-cycle (Susmel and Lazzarini, 2002) and in the medium-cycle fatigue regime (Lazzarini and Susmel; 2003). In particular, our method was seen to be capable of successfully accounting for the presence of both non-zero out of phase angles (Morrow, 1968; Lazzarini and Susmel; 2003; Susmel et al, 2005 ) and non-zero mean stresses (Susmel and Lazzarini, 2002; Lazzarini and Susmel; 2003; Susmel et al, 2005).

To understand how to use the MWCM in conjunction with the TCD, it is useful to remember here that Susmel and Taylor (2003) have observed that MWCM and PM are equivalent when they are employed to predict notch fatigue limits under fully-reversed uniaxial fatigue loading, provided that the MWCM is applied considering the stress state at the centre of the structural volume.

According to the arguments summarised above, it is possible to say that fatigue limits have to be estimated by considering a stress state which is representative of the entire stress field damaging the fatigue process zone. In particular, using the PM argument, it is possible to assume that the linear-elastic stress state calculated at the centre of the structural volume supplies all of the engineering information needed to perform an accurate high-cycle fatigue assessment. Moreover, in fatigue limit conditions, and independently of the stress concentration feature, the crack initiation phenomenon can be assumed to be governed by a Stage 1-like process. According to this, fatigue damage is more severe on the plane experiencing the maximum shear stress amplitude, and its amount depends on both $\tau_{a}$ and $\sigma_{n, \max }$ (Susmel, 2004).

\section{The Procedure to Apply the MWCM in Terms of the TCD for Fretting Fatigue}

A fretted component subjected to a system of external contact forces $(P$ and $Q)$ and also experiencing a bulk fatigue stress $\left(\sigma_{B}\right)$ gives rise to a subsurface multiaxial stress field within the contact region (Fig. 3). Further, as in components containing geometrical discontinuities the fretting regime is characterized by the presence a stress concentration at the contact surface which rapidly decays. This suggests that a threshold for crack initiation can be established using methodologies similar to those employed in notched components. In this setting, we seek to apply the MWCM in terms of TCD for fretting fatigue. To carry out the analysis it is necessary to determine the radius of the structural volume. It must always be calculated using fatigue properties (that is, $\Delta \sigma_{-1}$ and $\Delta K_{t h}$ ) determined under a load ratio, $\mathrm{R}$, equal to -1 (Susmel, 2004). In fact, as said above, the reference configuration is the one given by a 
through-cracked plate under fully-reversed uniaxial fatigue loading (Fig. 1a) and the presence of non-zero mean stresses as well as of non-zero out-of-phase angles is directly accounted for by Eq. (4) (Susmel, 2004; Susmel and Lazzarini, 2002; Lazzarini and Susmel; 2003; Susmel et al, 2005).

As far as the material solicitation is concerned the contact loads associated with the bulk fatigue stress provokes a non-proportional and complex (multiaxial) stress field. The stress field generated by $P$ and $Q(t)$ can be obtained in closed form for moderate values of the bulk stress. Somehow they generate a notch analogue problem as the contact stress field is high at the contact interface but is extremely localized. The procedure to compute the stress tensor at any interior material point for this configuration is a well established technique (Hills and Nowell, 1994) but, for sake of clarity more details will be provided in the next section.

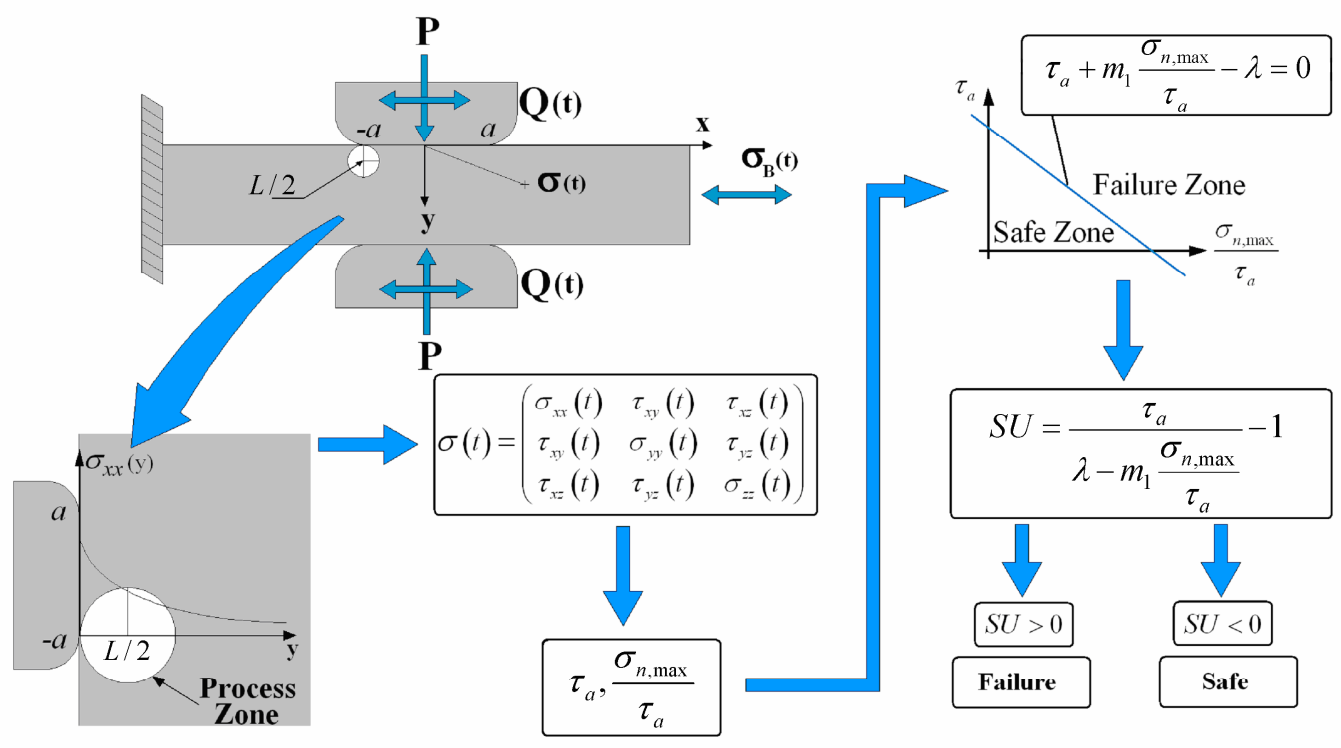

Figure 3. The procedure to apply the MWCM in terms of the TCD for fretting fatigue.

It is useful to remember that in real joints some localized plasticity may be provoked by the stress concentration present at the contact interface and a more rigorous analysis to determine the stress field should consider an appropriate constitutive model, which could account for the redistribution of stresses under the stress raiser. However, the use of our method is based on linear-elastic solutions. When the stress tensor is entirely defined during the load cycle and at the center of the structural volume $(y / a=L / 2 a)$, it is possible to determine the critical plane orientation as well as the shear stress amplitude and the maximum normal stress relative to such a plane by using the appropriate algorithms (Papadopoulos, 1998). Unfortunately, dealing with complex periodic load histories, this calculation is definitively time-consuming. Finally, if the condition expressed by eq. (4) is assured, then the studied component is in the fatigue limit condition. Figure 3 depicts an schematic view of the application of the proposed methodology to a cylinder on flat contact configuration under fretting conditions. In general, analytical approaches are not adequate to calculate the stress state at the centre of the structural volume for real mechanical assemblies. For this reason, engineers engaged in practical problems prefer to determine such stress states by post-processing linearelastic FE results. The proposed methodology is suitable for being used in conjunction with linear-elastic FE results, with the advantage that one does not have to define parameters such as nominal stress, equivalent stress intensity, etc.

\section{Available Experimental Data for Cylindrical Contacts}

The analysis will be validated against available experimental data for a cylinder on plane contact configuration (Fig 4a). These experiments have been reported in details elsewhere (Araujo et al,
2004; Nowell and Hills, 1987), hence only the basic information necessary to carry out the analysis will be provided here. The load history is depicted in Fig. 4b. A constant normal load, $P$, was applied to the fretting pads and held constant. A sinusoidal bulk load $B(t)$ applied to "the dog bone tensile" specimen induced (i) a bulk fatigue stress, $\sigma_{B}(t)=\sigma_{B \max } \sin (w t)$, and (ii) a in-phase shear load, $Q(t)=Q \max \sin (w t)$, where $\sigma_{B \max }$ and $Q_{\max }$ are the amplitudes of bulk stress and shear load, respectively, $w$ is the load frequency and $t$ is the time. Tests were designed to run in a partial slip regime, i.e. $Q<f P$, being $f=0.75$ the reported friction coefficient for the slip zones. It is worth of noticing that as the bulk load imposes a strain to the specimen the springs, which hold the fretting pads will also deform and therefore two parallel and opposite (to the bulk load) shear forces will develop (Figure 4). The value of shear forces obtained can be adjusted by varying the stiffness of the springs.

Table 1. Experimental parameters and critical contact size range.

\begin{tabular}{ccccc}
\hline Series & $p_{0}(\mathrm{MPa})$ & $Q_{\max } / P$ & $\sigma_{B \max }(\mathrm{MPa})$ & $a_{\text {crit }}(\mathrm{mm})$ \\
\hline 1 & 157 & 0.45 & 92.7 & $0.28-0.38$ \\
2 & 143 & 0.45 & 92.7 & $0.18-0.27$ \\
3 & 143 & 0.45 & 77.2 & $0.36-0.54$ \\
4 & 120 & 0.45 & 61.8 & $0.57-0.71$ \\
\hline
\end{tabular}

Four series of tests were considered. Within each series an average of eight tests using different pad radii were performed. Although the pad radius changed the superficial stress field was the same for all tests in a series (but the rate of stress decay varied). 
This provoked a size effect where tests containing larger contact widths (or pad radii) failed while for smaller contacts the tests run up to $10^{7}$ cycles (here considered as infinite life) before being interrupted. The range defined by the largest contact size to show infinite life and the smallest that failed was termed critical contact size range, $a_{c r i t}$. Table 1 reports the relevant load parameters and $a_{c r i t}$ for each data series. Pad radii and the number of cycles to fail are reported in Tables 2-5. Specimen dimensions are depicted in Fig. 5. Pads and specimens were made of an $\mathrm{A} 14 \% \mathrm{Cu}$ alloy (Young's modulus, $E=74 \mathrm{GPa}$, yield stress $\sigma_{y s}=465 \mathrm{MPa}$ and ultimate tensile strength, $\left.\sigma_{u s}=500 \mathrm{MPa}\right)$. In the broken specimens cracks initiated within the slip zone, at or close to the trailing edge of the contact.

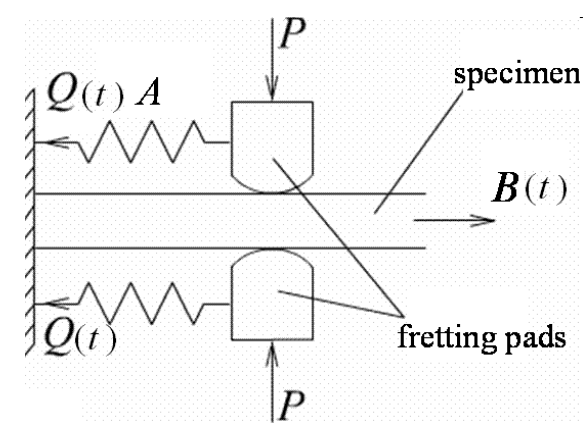

(a)

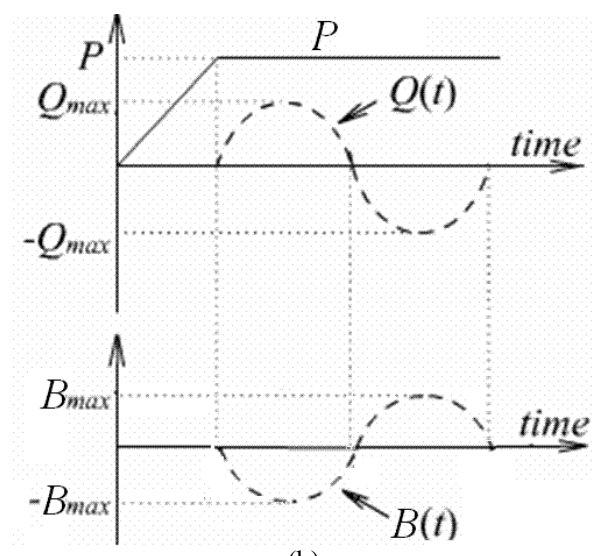

(b)

Figure 4. (a) Scheme of the cylinder on plane contact configuration tested and (b) load variation.

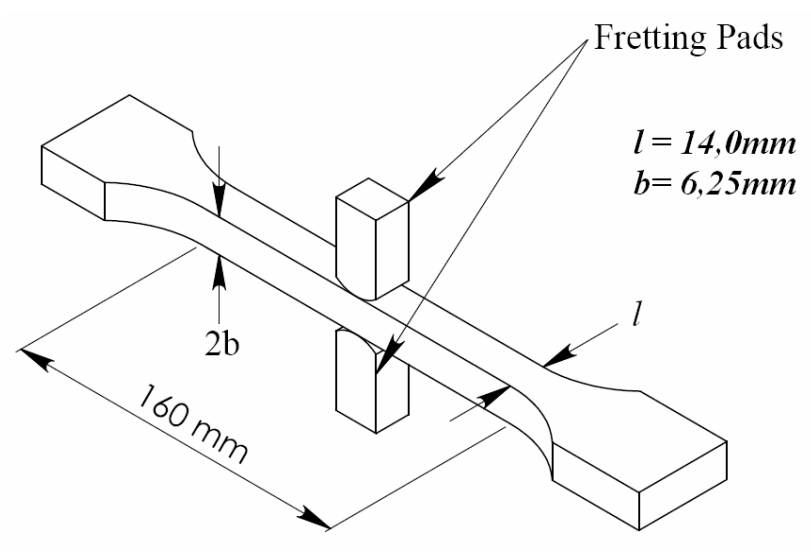

"Dog Bone" Tensile Specimen

Figure 5. Specimen dimensions.
Table 2. Data and error index provided by the application of the MWCM in terms of the CDM for Al series 1 data.

\begin{tabular}{|c|c|c|c|c|c|c|}
\hline \multicolumn{7}{|c|}{ Series 1 Data and Error Index } \\
\hline $\begin{array}{c}R \\
(\mathrm{~mm})\end{array}$ & $\begin{array}{c}a \\
(\mathrm{~mm})\end{array}$ & $\begin{array}{c}\text { Cycles } \\
\left(\mathrm{x} 10^{6}\right)\end{array}$ & $S U^{l}$ & $S U^{2}$ & $S U^{3}$ & $S U^{4}$ \\
\hline 12.5 & 0.10 & 10 & -0.0655 & -0.0730 & -0.0801 & -0.0758 \\
\hline 25 & 0.19 & 10 & 0.1504 & 0.1540 & 0.1574 & 0.1553 \\
\hline 37.5 & 0.28 & 10 & 0.2967 & 0.3039 & 0.3108 & 0.3066 \\
\hline 50 & 0.38 & 1.29 & 0.4087 & 0.4172 & 0.4255 & 0.4204 \\
\hline 75 & 0.57 & 0.67 & 0.5510 & 0.5585 & 0.5656 & 0.5612 \\
\hline 100 & 0.76 & 0.85 & 0.6384 & 0.6457 & 0.6527 & 0.6484 \\
\hline 125 & 0.95 & 0.73 & 0.6964 & 0.7047 & 0.7126 & 0.7077 \\
\hline 150 & 1.14 & 0.67 & 0.7519 & 0.7571 & 0.7621 & 0.7591 \\
\hline
\end{tabular}

Table 3. Data and error index provided by the application of the MWCM in terms of the CDM for Al series 2 data.

\begin{tabular}{|c|c|c|c|c|c|c|}
\hline \multicolumn{7}{|c|}{ Series 2 Data and Error Index } \\
\hline $\begin{array}{c}R \\
(\mathrm{~mm})\end{array}$ & $\begin{array}{c}a \\
(\mathrm{~mm})\end{array}$ & $\begin{array}{c}\text { Cycles } \\
\left(\mathrm{x} 10^{6}\right)\end{array}$ & $S U^{l}$ & $S U^{2}$ & $S U^{3}$ & $S U^{4}$ \\
\hline 12.5 & 0.09 & 10 & -0.1163 & -0.1239 & -0.1310 & -0.1267 \\
\hline 25 & 0.18 & 10 & 0.1023 & 0.1023 & 0.1023 & 0.1023 \\
\hline 37.5 & 0.27 & 4.04 & 0.2425 & 0.2466 & 0.2505 & 0.2481 \\
\hline 50 & 0.36 & 1.50 & 0.3388 & 0.3447 & 0.3504 & 0.3469 \\
\hline 75 & 0.54 & 0.80 & 0.4703 & 0.4758 & 0.4809 & 0.4778 \\
\hline 100 & 0.72 & 0.61 & 0.5514 & 0.5569 & 0.5623 & 0.5590 \\
\hline 125 & 0.90 & 1.24 & 0.6051 & 0.6118 & 0.6182 & 0.6143 \\
\hline 150 & 1.08 & 0.69 & 0.6567 & 0.6607 & 0.6644 & 0.6621 \\
\hline
\end{tabular}

Table 4. Data and error index provided by the application of the MWCM in terms of the CDM for Al series 3 data.

\begin{tabular}{|c|c|c|c|c|c|c|}
\hline \multicolumn{7}{|c|}{ Series 3 Data and Error Index } \\
\hline $\begin{array}{c}R \\
(\mathrm{~mm})\end{array}$ & $\begin{array}{c}a \\
(\mathrm{~mm})\end{array}$ & $\begin{array}{c}\text { Cycles } \\
\left(\mathrm{x} 10^{6}\right)\end{array}$ & $S U^{l}$ & $S U^{2}$ & $S U^{3}$ & $S U^{4}$ \\
\hline 12.5 & 0.09 & 10 & -0.2343 & -0.2409 & -0.2471 & -0.2433 \\
\hline 25 & 0.18 & 10 & -0.0284 & -0.0253 & -0.0224 & -0.0242 \\
\hline 50 & 0.36 & 10 & 0.2073 & 0.2146 & 0.2216 & 0.2173 \\
\hline 75 & 0.54 & 1.20 & 0.3391 & 0.3450 & 0.3506 & 0.3471 \\
\hline 100 & 0.72 & 1.42 & 0.4092 & 0.4182 & 0.4269 & 0.4216 \\
\hline 125 & 0.90 & 1.02 & 0.4762 & 0.4819 & 0.4874 & 0.4840 \\
\hline
\end{tabular}

Table 5. Data and error index provided by the application of the MWCM in terms of the CDM for Al series 4 data.

\begin{tabular}{|c|c|c|c|c|c|c|}
\hline \multicolumn{7}{|c|}{ Series 4 Data and Error Index } \\
\hline $\begin{array}{c}R \\
(\mathrm{~mm})\end{array}$ & $\begin{array}{c}a \\
(\mathrm{~mm})\end{array}$ & $\begin{array}{c}\text { Cycles } \\
\left(\mathrm{x} 10^{6}\right)\end{array}$ & $S U^{l}$ & $S U^{2}$ & $S U^{3}$ & $S U^{4}$ \\
\hline 25 & 0.14 & 10 & -0.2806 & -0.2793 & -0.2780 & -0.2788 \\
\hline 37.5 & 0.21 & 10 & -0.1655 & -0.1612 & -0.1571 & -0.1596 \\
\hline 50 & 0.28 & 10 & -0.0837 & -0.0776 & -0.0718 & -0.0754 \\
\hline 75 & 0.42 & 10 & 0.0319 & 0.0377 & 0.0432 & 0.0398 \\
\hline 100 & 0.57 & 10 & 0.1112 & 0.1164 & 0.1213 & 0.1183 \\
\hline 125 & 0.71 & 1.57 & 0.1613 & 0.1667 & 0.1719 & 0.1687 \\
\hline 150 & 0.85 & 1.23 & 0.1972 & 0.2035 & 0.2094 & 0.2058 \\
\hline
\end{tabular}

The configuration tested is particularly interesting to analysis as it provides a closed form solution for the cyclic stress field. The direct and shear tractions are defined by the Hertz (1982) and Mindlin (1949) solutions. These can be used with Muskhelishivili's (1953) potentials to determine the stress components associated with the normal and shear loads. 
Finally superposition is considered to compute the resultant stress tensor, so that at the instants of maximum and minimum bulk/shear loads it will be given by:

$$
\begin{aligned}
& \frac{\boldsymbol{\sigma}(y, x, t)}{p_{o}}=\left(\frac{\boldsymbol{\sigma}^{n}\left(\frac{x}{a}, \frac{y}{a}\right)}{p_{0}}\right) \pm f\left(\frac{\boldsymbol{\sigma}^{t}\left(\frac{x}{a}, \frac{y}{a}\right)}{f p_{0}}\right) \mp \\
& \mp f \frac{c}{a}\left(\frac{\boldsymbol{\sigma}^{t}\left(\frac{x-e}{c}, \frac{y}{c}\right)}{f p_{0}}\right)+\frac{\boldsymbol{\sigma}_{B}(t)}{p_{0}}
\end{aligned}
$$

being the combination of signs + and - for the maximum load step. During loading or unloading:

$$
\begin{aligned}
& \frac{\boldsymbol{\sigma}(y, x, t)}{p_{o}}=\left(\frac{\boldsymbol{\sigma}^{n}\left(\frac{x}{a}, \frac{y}{a}\right)}{p_{0}}\right) \pm f\left(\frac{\boldsymbol{\sigma}^{t}\left(\frac{x}{a}, \frac{y}{a}\right)}{f p_{0}}\right) \mp \\
& \mp 2 f \frac{d(t)}{a}\left(\frac{\boldsymbol{\sigma}^{t}\left(\frac{x-e^{\prime}(t)}{d(t)}, \frac{y}{d(t)}\right)}{f p_{0}}\right) \pm f \frac{c}{a}\left(\frac{\boldsymbol{\sigma}^{t}\left(\frac{x-e}{c}, \frac{y}{c}\right)}{f p_{0}}\right)+\frac{\boldsymbol{\sigma}_{B}(t)}{p_{o}}
\end{aligned}
$$

For unloading conditions the correct sequence of signs to be considered in eq. (6) is,-+ and - . Here $p_{o}$ is the peak pressure, $c$ and $e$ are the stick zone half width and its offset from the centre of the contact at the instant of maximum or minimum shear load. At

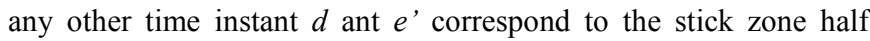
width and its offset from the centre of the contact. The superscripts $n$ and $t$ stand for the stress components due to the normal and tangential loads, respectively. $\sigma_{B}(t)$ is the stress tensor associated with the bulk fatigue load, hence $\sigma_{x x}$ is its unique stress component different from zero. Plane strain conditions are assumed. Explicit expressions to compute $c, e, d, e^{\prime}, \sigma^{n}$ and $\sigma^{t}$ are given in a convenient form by Hills and Nowell (1994).

\section{Results}

The MWCM will now be applied at the center of the structural volume in order to estimate the fretting fatigue limit for the experimental configuration considered in this work. Hence the first step in the analysis is to define the material parameter $L$. Susmel et al. (2004) reported $L=0.1 \mathrm{~mm}$ for an $\mathrm{Al} 4 \% \mathrm{Cu}$ alloy having the same fatigue limit $\left(\Delta \sigma_{-l}=248 \mathrm{MPa}\right)$ as the one tested by Nowell under fretting. The center of the the structural volume is then $L / 2=0.05 \mathrm{~mm}$. At this depth and at the trailing edge of the the contact zone (hot spot), i.e. $x / a=-1$, the cyclic stress tensor was analytically extracted at twelve different load steps by using eqs. (11) and (12). To continue the analysis the $S U$ index (eq. 10) needs to be computed from the stress history defined at this material point. Its calculation also requires two fatigue parameters at different loading conditions, such as the fatigue limits for fully reversed bending and torsion or, alternativelly, the fatigue limits for stress ratios $R=-1$ and $R=0$. In a recent work on the effects of mean normal stresses over the fatigue limit for a number of alloys, Dowling (2004) reported that, among a number of models assessed, the one proposed by Smith et al (1970) provided the best estimates of the fatigue limit for Al alloys tested under different $R$ ratios. However, as mentioned before, the models of Goodman and Morrow will also be used to isllustrate the effect of estimating the unknow fatigue parameter on the predictive methodology. The fatigue limits for $R=0$ estimated by SWT (eq. 6), Goodman (eq. 7) and Morrow (considering $\tilde{\sigma}_{f B}=635 \mathrm{MPa}$ (eq. 8) and ${\sigma^{\prime}}_{f}=1015 \mathrm{MPa}$ (eq. 9)) were 87.7, 99.4, 130.7 and $110.5 \mathrm{MPa}$, respectivelly. The error index values calculated by considering each of these estimates and applying the MWCM in terms of CDM to the available experimental data were termed $S U^{l}, S U^{2}, S U^{3}$ and $S U^{4}$. Such results are reported in Tables 2 to 5. It is also reported in these tables information concerning the pad radius, $R$, the contact semiwidth, $a$ and the number of cycles in which the specimen failed for all tests. Tests that achieved $10^{7}$ cycles were stopped and further investigation did not reveal the presence of cracks within the fretted zones or elsewhere within the contact region. At this stage the reader should be reminded that negative $S U$ values mean that the solicitation is below the limit established by the multiaxial criterion and hence no failure is expected. In Table 6 these results are summarised.

Tabela 6. Error Index results for tests where the predictive methodology do not agree with data.

\begin{tabular}{|c|c|c|c|c|c|c|c|}
\hline Series & $\begin{array}{c}R \\
(\mathrm{~mm})\end{array}$ & $\begin{array}{c}a \\
(\mathrm{~mm})\end{array}$ & $\begin{array}{c}\text { Cycles } \\
\left(\mathrm{x} 10^{6}\right)\end{array}$ & $S U^{l}$ & $S U^{2}$ & $S U^{3}$ & $S U^{4}$ \\
\hline \multirow{2}{*}{1} & 25 & 0.19 & 10 & 0.1504 & 0.1540 & 0.1574 & 0.1553 \\
\cline { 2 - 8 } & 37.5 & 0.28 & 10 & 0.2967 & 0.3039 & 0.3108 & 0.3066 \\
\hline 2 & 25 & 0.18 & 10 & 0.1023 & 0.1023 & 0.1023 & 0.1023 \\
\hline 3 & 50 & 0.36 & 10 & 0.2073 & 0.2146 & 0.2216 & 0.2173 \\
\hline \multirow{2}{*}{4} & 75 & 0.42 & 10 & 0.0319 & 0.0377 & 0.0432 & 0.0398 \\
\cline { 2 - 8 } & 100 & 0.57 & 10 & 0.1112 & 0.1164 & 0.1213 & 0.1183 \\
\hline
\end{tabular}

It reports all cases where the estimated fretting limits diverge from experimental data. It can be noticed that the estimates provided for the twenty nine tests of the four different experimental series considered do not agree with data in six cases only, independently of the index used $\left(S U^{1}, S U^{2}, S U^{3}\right.$ or $\left.S U^{4}\right)$. Furthermore, the incorrect predictions were all in the conservative side, i.e., they indicated that the specimens would failure while infinite lives were achieved. For instance, in the $\mathrm{Al}$ series 4 data the largest contact size to show infinite life was $a=0.57 \mathrm{~mm}$, while $S U^{l}=0.1112$ means that failure is expected. The level of conservatism involved in the analysis is always raised if the estimates are based on $S U^{2}$ or $S U^{3}$ or $S U^{4}$. Again considereing Al series 4, this can be clearly verified for the test whose $a=0.42 \mathrm{~mm}$. In this case $S U^{l}=0.0319$ (Table 5) is the smallest positive index computed for this contact size. $S U^{2}, S U^{3}$ and $S U^{4}$ present larger positive values of the index than $S U^{I}$ for this same test. It is worth of note that a larger positive index indicates an earlier failure of the specimen, however for this test no failure was observed.

Another interesting way to illustrate the results provided by the analysis is to plot the experimental data on a $\tau_{a} x \sigma_{n, \max } / \tau_{a}$ stress space, which also contains the failure lines defined by considering the use of $S U^{l}, S U^{2}, S U^{3}$, and $S U^{4}$.

Fig. 6 depicts such diagram for Al series 1 (Fig. 6(a)), 2 (Fig. 6(b)), 3 (Fig. 6(c)) and 4(Fig. 6(d)). It is apparent that the line defined by considering $S U^{l}$ defines a region in the stress space which can take larger values of $\tau_{a}$ for intermediate ratios $\left(\sigma_{n, \max } / \tau_{a}\right.$ $<1)$ than the other indexes. As all the tests for the four data series fall within the region where $\sigma_{n, \max } / \tau_{a}<1$, this explains why the estimates provided by $S U^{l}$ are always the least conservative. 

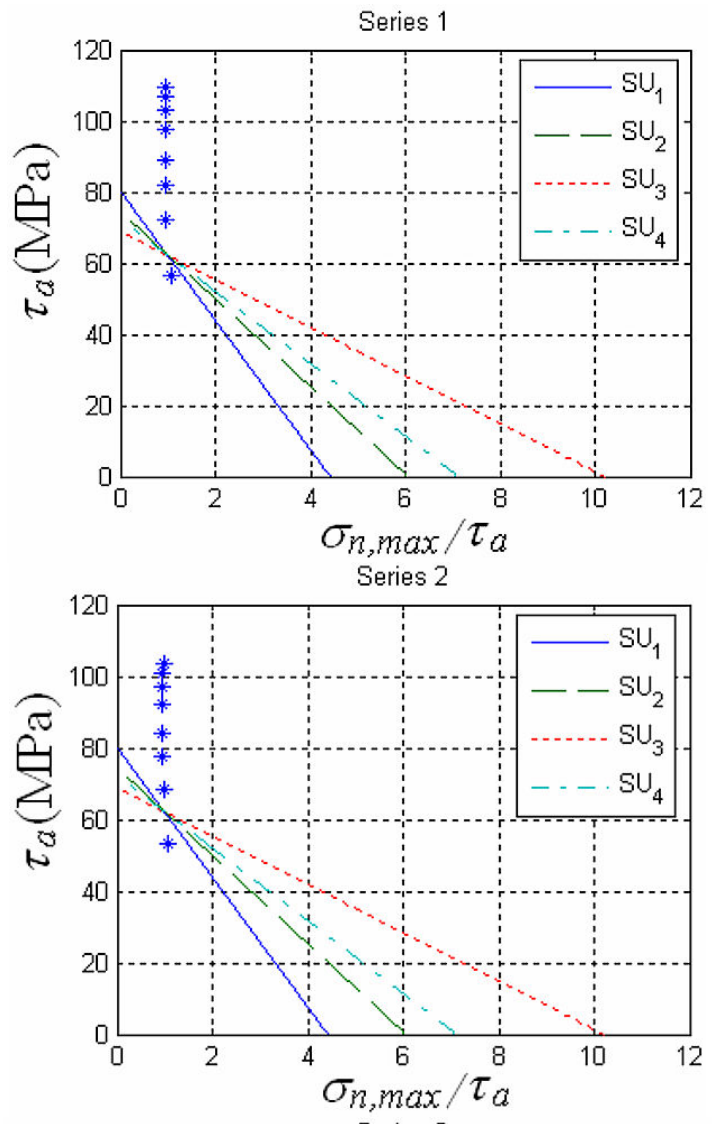

Series 3
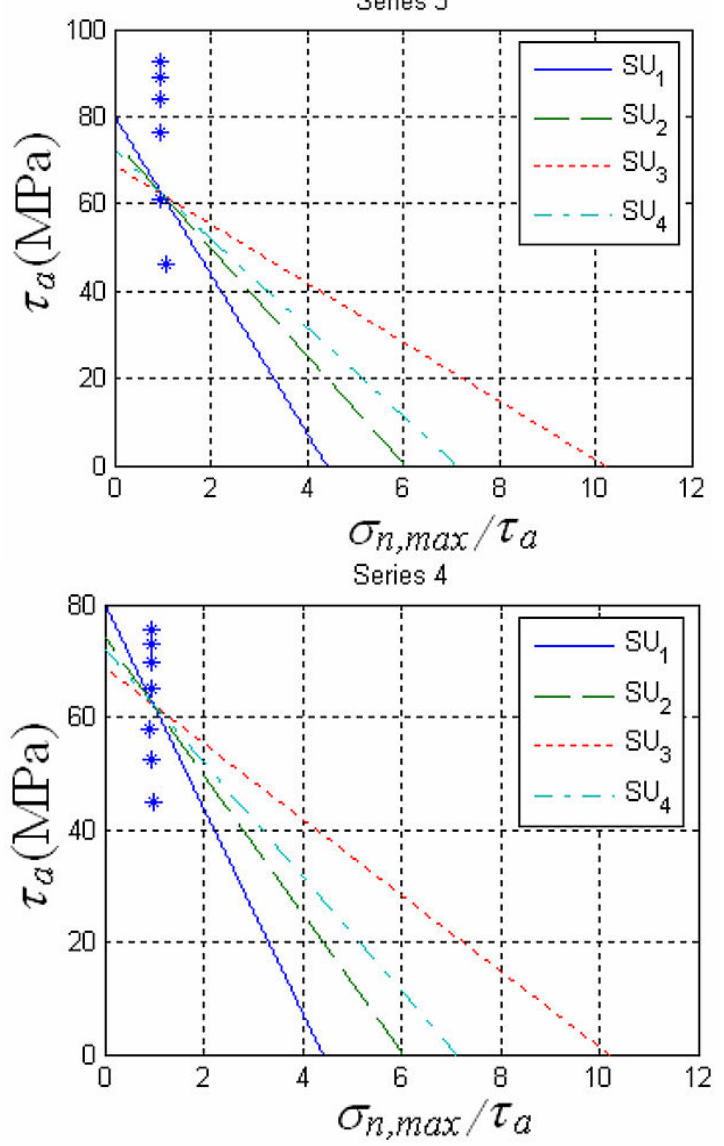

Figure 6: $\tau_{a} \times \sigma_{n, \max } / \tau_{a}$ stress space for Al series 1, 2, 3 and 4 data and failure lines considering the use of $\mathrm{SU}^{1}, \mathrm{SU}^{2}, \mathrm{SU}^{3}$, and $\mathrm{SU}^{4}$.

\section{Conclusions}

A fretting crack initiation threshold methodology was established. It is based on the application of the Modified Wöhler Curves Method and on the Stress Point Concept. The methodology presented successful estimates in twenty three of the twenty nine fretting fatigue tests considered in this work. Further, for the cases where the methodology failed it predicted crack initiation while the specimens last forever, i.e. it was conservative. Compared with other notch analogue methodologies proposed by the authors (Araujo et al, 2006) for fretting fatigue, this approach has the advantage of defining the critical distance as a material parameter. Hence, if the basic fatigue parameters are appropriately defined for a specific alloy the crack initiation risk can be directly computed without the need to carry out further fretting fatigue calibration tests to define the size of the structural volume. The methodology proved simple to implement and the fact that it requires only the linearelastic stress state calculated at the centre of the structural volume to perform an accurate high-cycle fatigue assessment makes it extremely appealing from a engineering point of view. On the other hand, it must be stated that further validation of the proposed approach considering different materials and contact configuration have to be carried out before using it to design real components. For the tests considered in this work it was observed that the estimation of the fatigue limit under zero to tension loading by the SWT parameter provided the best results. The objective of this comparative analysis was just to illustrate that the estimation of fatigue properties had a clear effect on the predictive methodology. In this way, researchers involved in this field could be warned and feel encouraged to carry out a more complete fatigue characterization of the material before it was fretted. Information such as the Mode I treshold stress intensity and the fatigue limit under different loading conditions would provide the means to evaluate more precisely a number of predictive methodologies based on notch and multiaxial fatigue theories. As usual, the basic mechanical properties and information concerning the material grain size, heat/chemical treatments, etc should also be provided.

\section{References}

Akiniwa, Y., Tanaka, K., Kimura, H., 2001, Microstructural effects on crack closure and propagation thresholds of small fatigue cracks. Fatigue Fract. Engng. Mater. Struct., Vol.24, pp. 817-831.

Araújo J. A., Vivacqua R. C., Bernardo A. T. S., Mamiya E. N., 2006, A crack initiation threshold methodology in fretting fatigue. Journal of Strain Analysis, Vol. 41, No 5, pp. 363-368.

Araújo, J.A., and Nowell, D., 1999, Analysis of pad size effects in fretting fatigue using short crack arrest methodologies, International Journal of Fatigue, Vol. 21, No 9, pp. 947-956.

Araújo, J.A., Nowell, D. and Vivacqua, R. C., 2004, The use of multiaxial fatigue models to predict fretting fatigue life of components subjected to different contact stress fields, Fatigue Fract. Engng. Mater. Struct., Vol.27, pp. 967-978.

Dowling, N., 2004, Mean Stress Effects in Stress-Life and Strain-Life Fatigue, 2nd SAE Brasil International Conference on Fatigue, São Paulo, 2223th June.

Fouvry, S., Elleuch, K., and Simeor, G., 2002, Prediction of crack nucleation under partial slip fretting conditions, Journal of Strain Analysis, Vol. 37, No 6, pp 549-564.

Goodman, J., 1919, Mechanics Applied to Engineering, Longmans, Green and Co., London, pp. 631-636.

Hertz, H., 1882, Über die Berührung fester elastischer Körper, Jnl. Reine und angewandte Mathematik, Vol.92, pp. 156-171.

Hills, D. A. and Nowell, D., Mechanics of fretting fatigue, Kluwer Academic publishers, Dordrecht, 1994.

Lazzarin, P., Susmel, L., 2003, A Stress-Based Method to Predict Lifetime under Multiaxial Fatigue Loadings. In: Fatigue Fract. Engng. Mater. Struct., Vol.26, pp. 1171-1187.

Menghetti, G., Susmel, L., Tovo R., 2004, High cycle fatigue crack paths in specimens having different stress concentration features. In: F. 
Nilsson (Ed.), Proc. of 15th European Conference of Fracture, Stockholm, Sweden.

Miller, K. J., 1993, The two thresholds of fatigue behaviour. In: Fatigue Fract. Engng. Mater. Struct., Vol.16, pp. 931-939.

Mindlin, R. D., 1949, Compliance of elastic bodies in contact, Jnl. App. Mech., Vol.16, pp. 259-268.

Morrow, J., 1964, "Fatigue Properties of Metals," Section 3.2 of Fatigue Design Handbook, Pub. No. AE-4, Society of Automotive Engineers, Warrendale, PA, 1968. Section 3.2 is a summary of a paper presented at a meeting of Division 4 of the SAE Iron and Steel Tech. Committee, Nov. $4^{\text {th }}$.

Muskhelishvili, N. I., 1953, Some Basic Problems of Mathematical Theory of Elasticity, Noordhoff, Gröningen.

Nowell, D., and Hills, D.A., 1987, Mechanics of fretting fatigue tests, Int. J. Mech. Sci., Vol.29, pp. 355

Papadopoulos, I. V., 1998, Critical Plane Approaches in High-Cycle Fatigue: on the Definition of the Amplitude and Mean Value of the Shear Stress Acting on the Critical Plane. Fatigue Fract. Engng. Mater. Struct., Vol.21, pp. 269-285.

Smith, K.N., Watson, P., Topper, T.H., 1970, A Stress-Strain Function for the Fatigue of Metals, J. Mater., Vol. 5, No. 4, pp. 767-778.

Susmel, L., 2004, A unifying approach to estimate the high-cycle fatigue strength of notched components subjected to both uniaxial and multiaxial cyclic loadings. Fatigue Fract. Engng. Mater. Struct., Vol.27, pp. 391-411.

Susmel, L., Atzori, B., Meneguetti, G., 2004, Material fatigue properties for assessing mechancial components weakened by notches and defects, Fatigue and Fracture of Engng. Mater. Struct., Vol.27, pp. 1-15.

Susmel, L., Lazzarin, P., 2002, A bi-parametric Wöhler curve for high cycle multiaxial fatigue assessment. Fatigue Fract. Engng. Mater. Struct., Vol.25, pp. 63-78.
Susmel L., Taylor, D., 2003, Two methods for predicting the multiaxial fatigue limits of sharp notches. Fatigue Fract. Engng. Mater. Struct., Vol.26, pp. 821-833.

Susmel, L., Taylor, D., 2004a, Fatigue Design in the Presence of Stress Concentrations. In: Int. J. of Strain Analysis for Eng. Components, Vol. 385, pp. $443-452$

Susmel, L., Taylor, D., 2004b, Non-propagating cracks under in-phase mode I and II loadings. Submitted to: Fatigue Fract. Engng. Mater. Struct.

Susmel, L., Taylor, D., Tovo, R., 2003, Crack paths in sharply notched specimens under in-phase biaxial loadings. In: A. Carpinteri and L. Pook (Ed.), Int. Conf. on Crack Paths, Parma, Italy.

Susmel L., Tovo R., Lazzarin P., 2005, The mean stress effect on the high-cycle fatigue strength from a multiaxial fatigue point of view. Int. J. Fatigue, Vol.27, pp. 928-943.

Tada, H., Paris, C. P.; Irwin, R. G., 2000, The Stress Analysis of Cracks - Handbook. Professional Engineering Publishing, Bury St. Edmunds, UK ( $3^{\text {rd }}$ Edition).

Taylor, D., 1999, Geometrical effects in fatigue: a unifying theoretical model. Int. J. Fatigue, Vol.21, pp. 413-420.

Taylor, D., 2001, A mechanistic approach to critical-distance methods in notch fatigue. Fatigue Fract. Engng. Mater. Struct., Vol.24, pp. 215-224.

Vallelano C., Dominguez, J., Navarro A., 2004, Predicting the fretting fatigue limit for spherical contact. Engineering Failure Analysis, Vol.11, pp. 727-736.

Yates, J. R., Brown, J. R., 1987, Prediction of the length of nonpropagating fatigue cracks. In: Fatigue Fract. Engng. Mater. Struct., Vol.10, pp. 187-201. 